\title{
ORIGINAL ARTICLE \\ Potential and limits to unravel the genetic architecture and predict the variation of Fusarium head blight resistance in European winter wheat (Triticum aestivum L.)
}

\author{
Y Jiang ${ }^{1}$, Y Zhao ${ }^{1}$, B Rodemann ${ }^{2}$, J Plieske ${ }^{3}$, S Kollers ${ }^{4}$, V Korzun ${ }^{4}$, E Ebmeyer ${ }^{4}$, O Argillier ${ }^{5}$, M Hinze $^{6}$, \\ J Ling $^{1}$, MS Röder ${ }^{1}$, MW Ganal ${ }^{3}$, MF Mette ${ }^{1}$ and JC Reif ${ }^{1}$
}

Genome-wide mapping approaches in diverse populations are powerful tools to unravel the genetic architecture of complex traits. The main goals of our study were to investigate the potential and limits to unravel the genetic architecture and to identify the factors determining the accuracy of prediction of the genotypic variation of Fusarium head blight (FHB) resistance in wheat (Triticum aestivum L.) based on data collected with a diverse panel of 372 European varieties. The wheat lines were phenotyped in multi-location field trials for FHB resistance and genotyped with 782 simple sequence repeat (SSR) markers, and 9k and 90k single-nucleotide polymorphism (SNP) arrays. We applied genome-wide association mapping in combination with fivefold crossvalidations and observed surprisingly high accuracies of prediction for marker-assisted selection based on the detected quantitative trait loci (QTLs). Using a random sample of markers not selected for marker-trait associations revealed only a slight decrease in prediction accuracy compared with marker-based selection exploiting the QTL information. The same picture was confirmed in a simulation study, suggesting that relatedness is a main driver of the accuracy of prediction in marker-assisted selection of FHB resistance. When the accuracy of prediction of three genomic selection models was contrasted for the three marker data sets, no significant differences in accuracies among marker platforms and genomic selection models were observed. Marker density impacted the accuracy of prediction only marginally. Consequently, genomic selection of FHB resistance can be implemented most cost-efficiently based on low- to medium-density SNP arrays.

Heredity (2015) 114, 318-326; doi:10.1038/hdy.2014.104; published online 12 November 2014

\section{INTRODUCTION}

Fusarium head blight (FHB) severely impacts wheat production worldwide (Buerstmayr et al., 2009). FHB disease is mainly caused by the fungal pathogens Fusarium graminearum and Fusarium culmorum, which attack the spikes of wheat, leading to shriveled and discolored kernels containing mycotoxins (Pestka and Smolinski, 2005). The European Union and other international legislative bodies rigorously limit the tolerated mycotoxin content in wheat to be used as food or feed, making infected wheat often unmarketable (Verstraete, 2008).

Breeding for FHB resistance can reduce the detrimental effects on wheat grain yield and quality. However, reliable phenotypic selection requires labor intensive and thus costly artificial inoculation to guarantee homogeneous disease pressure in field trials (Miedaner et al., 2002). As an alternative approach, marker-assisted selection has been promoted in breeding for decreased FHB susceptibility in wheat varieties (Miedaner et al., 2009). Large effect quantitative trait locus (QTL) such as Fhb1 and Fhb5 were identified in crosses with the exotic Sumai 3 and CM82036 donor lines (Becher et al., 2013). However, despite worldwide efforts, the frequency of favorable Fhb1 and Fhb5 alleles is still low in European and US elite wheat germplasm (Sneller et al., 2010; Bernardo et al., 2012; Miedaner and Korzun, 2012). One reason is the long time need to reduce linkage drag associated with marker-based introgression of Fhb1 or Fhb5 (Miedaner and Korzun, 2012). In contrast to $F h b 1$ and $F h b 5$, the effects of FHB resistance QTL that were identified in adapted European or US wheat germplasm and thus are not associated with linkage drag are only rather small (Buerstmayr et al., 2008; Löffler et al., 2009; Sneller et al., 2010). This so far hinders an efficient application of marker-assisted selection for FHB resistance for QTL alleles identified in adapted elite germplasm.

Genomic selection has been suggested as an attractive alternative to marker-assisted selection to predict phenotypes for traits that are controlled by multiple genes with small effects (Meuwissen et al., 2001). In contrast to marker-assisted selection, which predicts genotypic values based only on the markers associated with significant QTL detected in the genome-wide association mapping, genomic selection simultaneously utilizes large numbers of markers distributed across the genome to train a prediction model. The potential of genomic selection for predicting FHB resistance has been studied previously using 170 lines, which were phenotyped at the US

${ }^{1}$ Department of Cytogenetics and Genome Analysis, Leibniz Institute of Plant Genetics and Crop Plant Research (IPK), Gatersleben, Germany; ${ }^{2}$ Julius Kühn Institute, Braunschweig, Germany; ${ }^{3}$ TraitGenetics GmbH, Gatersleben, Germany; ${ }^{4}$ KWS Lochow GmbH, Bergen, Germany; ${ }^{5}$ Syngenta Seeds S.A.S., Orgerus, France and ${ }^{6}$ Syngenta Seeds $\mathrm{GmbH}$, Bad Salzuflen, Germany

Correspondence: Professor JC Reif, Department of Cytogenetics and Genome Analysis, Leibniz Institute of Plant Genetics and Crop Plant Research (IPK), 06466 Gatersleben, Germany.

E-mail: reif@ipk-gatersleben.de

Received 2 May 2014; revised 9 September 2014; accepted 9 October 2014; published online 12 November 2014 
cooperative FHB wheat nursery and genotyped with genome-wide diversity array technology as well as with functional markers for FHB resistance (Rutkoski et al., 2012). Despite the small number of genotypes, these findings suggested that US cooperative FHB nursery data can be useful for genomic selection.

Investigating the accuracy of prediction of genomic versus markerassisted selection in wheat revealed that genomic selection usually increases accuracy, with some dependence on the trait under consideration and the related linkage disequilibrium structure (Guo et al., 2012; Rutkoski et al., 2012; Miedaner et al., 2013; Zhao et al., 2013, 2014). One interesting finding was that for FHB resistance, as for some other complex traits, the differences between the mean accuracies of marker-assisted and genomic selection were surprisingly small (Rutkoski et al., 2012). A genome-wide mapping study in a large hybrid wheat population revealed that the accuracy of marker-assisted selection is not only depending on functional knowledge of the genetic architecture, but also profits from genetic relationships between individuals in the training and the validation population (Gowda et al., 2014). This could provide an explanation for the surprisingly low differences in mean accuracy of genomic and marker-assisted selection. Studies on the influence of relatedness versus functional QTL information as driving forces of the accuracy of prediction of marker-assisted selection, however, are lacking to the best of our knowledge.

Marker systems that have been used for genome-wide fingerprinting in wheat include restriction fragment length polymorphism (Chao et al., 1989), amplified fragment length polymorphism (Barrett and Kidwell, 1998), simple sequence repeat (SSR) markers (Röder et al., 1998), diversity array technology (Akbari et al., 2006), singlenucleotide polymorphism (SNP) markers (Cavanagh et al., 2013), and most recently genotyping-by-sequencing approaches (Poland et al., 2012). When Heslot et al. (2013) compared the accuracy of prediction of genomic selection based on genotyping-by-sequencing versus diversity array technology markers, they found advantages of genomic selection based on genotyping-by-sequencing data, which they interpreted to be mainly because of the higher number of markers. The same study also unraveled a sampling bias for the used diversity array technology markers. Würschum et al. (2013) compared SNP and SSR markers in wheat and found only low congruency among the genetic relatedness matrices estimated with the two marker types. As genetic relatedness is one of the major forces exploited in genomic selection, substantial differences are expected for the prediction models trained with SNP or SSR markers in wheat.

In this study, we draw on published data derived from a diversity panel consisting of 358 recent European winter wheat varieties plus 14 spring varieties, which had been phenotyped for FHB resistance and genotyped with 732 SSR markers (Kollers et al., 2013a, b). The 372 varieties were additionally genotyped with 9k (Cavanagh et al., 2013) and 90k SNP arrays (Wang et al., 2014a). Aiming to determine the genetic architecture of FHB resistance in the European wheat panel, we applied genome-wide association mapping, which detected the highest number of QTL when the analysis was based on the highdensity 90k SNP data. However, when tested in fivefold crossvalidation, marker-assisted selection using the detected QTL allowed only marginally higher accuracies of prediction than calculation based on random samples of markers not selected for marker-trait associations. Consistent with a simulation approach, this suggested relatedness as a main driver of the accuracy of prediction. We then set out to test the accuracy of prediction of FHB resistance by genomic selection and to identify the factors that determine accuracy. When three genomic selection models were contrasted for the three marker data sets, no significant differences in accuracies among marker platforms and genomic selection models were observed. Marker density impacted the accuracy of prediction only marginally, indicating that genomic selection of FHB resistance can be implemented most costefficiently based on low- to medium-density SNP arrays.

\section{MATERIALS AND METHODS}

\section{Plant material and phenotypic data analyses}

A total of 358 European winter wheat varieties plus 14 spring wheat varieties were used in this study (Kollers et al., 2013a, b). The 372 wheat varieties were evaluated for FHB resistance over 2 years in four environments in Germany in trials with three replications. In all environments, lines were artificially spray inoculated as described in detail by Kollers et al. (2013a). To compensate for different flowering times of the wheat lines, inoculation was carried out at three time points in order to inoculate each genotype at least once at full flowering. As a measure of FHB resistance, the FHB score with a possible range from $0 \%$ (most resistant) to $100 \%$ (most susceptible) was calculated as FHB score $=\mathrm{FHB}$ incidence $\times \mathrm{FHB}$ severity $/ 100 \%$, with FHB incidence representing the percentage of infected spikes in a test plot and FHB severity representing the mean percentage of infected area on infected spikes.

We performed a one-step phenotypic analysis using a linear mixed-model with effects for genotype, environment, interaction of genotype by environment, and replication. To estimate variance components all effects were assumed to be random. Broad-sense heritability $\left(h^{2}\right)$ was estimated on an entry-mean basis as $h^{2}=\sigma_{\mathrm{G}}^{2} /\left(\sigma_{\mathrm{G}}^{2}+\sigma_{\mathrm{GxE}}^{2} / E+\sigma_{\mathrm{e}}^{2} / R^{\star} E\right)$, where $E$ refers to the number of environments, $R$ is the number of replications, $\sigma_{\mathrm{GxE}}^{2}$ refers to the variance of the genotype by environment interactions and $\sigma^{2}{ }_{e}$ refers to the error variance. A second linear mixed model, in which genotypic effects were assumed to be fixed and all other effects remained random, was used to get the best linear unbiased estimates of FHB scores for each variety.

\section{Genotypic data}

The 372 varieties were genotyped with a 9k Infinium SNP array (Cavanagh et al., 2013; Supplementary Table S1), a 90k Infinium SNP array (Wang et al., 2014a) and 732 SSR markers resulting in 782 loci (Kollers et al., 2013a, b, 2014). In total, $78 \%$ of the SNP markers from the $9 \mathrm{k}$ array were also present on the $90 \mathrm{k}$ SNP array.

We performed quality control for SNP markers to exclude those with rates of missing values above $5 \%$, rates of heterozygotes above $5 \%$ and allele frequencies $<0.05$ or $>0.95$. For SSR markers, we excluded those with rates of missing value above $10 \%$ and rates of heterozygotes above $5 \%$. All varieties were additionally genotyped for three predefined functional markers, the dwarfing genes Rht-B1, Rht-D1 and the photoperiodism gene Ppd-D1 (Ellis et al., 2002; Beales et al., 2007), which were used for association mapping.

We estimated Rogers' distances for each pair of lines using the three different marker sets. Correlations between each pair of distance matrices were calculated and the Mantel test (Mantel, 1967) was applied. Principal component analyses were performed for the three marker sets. To test for differences in the proportion of variance explained by the individual principal components, we implemented a bootstrap approach as outlined in detail elsewhere (Heslot et al., 2013).

\section{Association mapping and marker-assisted selection}

We applied for each marker set a two-step association mapping scan. In the first step, the best linear unbiased estimate for each genotype in each environment was estimated assuming fixed genotype and random replication effects using a linear mixed model. Then, in the second step, a standard linear mixed-model approach (Yu et al., 2006) was used to perform a genome-wide association mapping scan:

$$
y=\mu+\alpha m+X g+E l+e,
$$

where $y$ is the vector of best linear unbiased estimates for each genotype in each environment, $\mu$ is the vector of common intercept term, $m$ is the effect of the marker being tested, $\alpha$ denotes the vector of scores of the marker, $g$ is the vector of genotype effects, $l$ is the vector of environment effects, $X$ and $E$ are the corresponding design matrices, and $e$ is the residual term. In the model only 
the marker effect was assumed to be fixed and all other effects are random. The population structure was considered by assuming $g \sim N\left(0, G \sigma_{G}^{2}\right)$, where $G$ is twice the kinship matrix estimated as 1 minus the Rogers' distances and $\sigma_{G}^{2}$ is the genotypic variance estimated by a restricted maximum likelihood approach. To check whether the model is able to adequately control the population or family structure, a qq-plot was drawn based on the observed $P$-values and expected $P$-values of all markers (Yu et al., 2006). Significance of marker-trait associations was tested based on the Wald F statistic. The proportion of the phenotypic variance explained by all QTLs was estimated using the $R^{2}$ values fitting a multiple regression (Utz et al., 2000), which ignores information of relatedness. In addition, we implemented the method suggested by Sun et al. (2010) and estimated the likelihood-ratio-based $R^{2}$ values $\left(R_{L R}^{2}\right)$, which takes the information of relatedness into account.

Given the same threshold, there would be more marker-trait associations detected in the 90k SNP array than in the 9k SNP array. However, the number of informative markers is not necessarily higher in the 90k array because of possible linkage disequilibrium. We defined a new statistic parameter called effective number of marker-trait associations. To calculate it, we first performed principal component analysis with the significant markers among the 372 lines, and then extracted the minimal number of principal components needed to portray $95 \%$ of the total variation.

SNP markers were scored as 2,1 or 0 as the number of copies of a particular allele. SSR markers were treated differently from SNP markers. For the SSR markers, we avoided treating each allele as an individual locus. Instead, markers were scored as a class (factor) indicating the presence of the different alleles. However, SSR markers that could reliably be mapped to different genomic positions were considered as separate loci.

The accuracy of prediction of marker-assisted selection was evaluated by fivefold cross-validation with a total of 100 cross-validation runs. In each run, four-fifths of the 372 wheat lines used in the study were assigned to the estimation set, whereas the remaining one-fifth was assigned to the test set. We then performed an association mapping scan for the estimation set and recorded the detected QTLs. We applied three different significance thresholds to study their influence on the accuracy of prediction. For the 9k SNP array and SSR markers, we set the thresholds to $P<0.01,0.005$ and 0.0001 . For the $90 \mathrm{k}$ SNP array, the thresholds were $P<0.005,0.001$ and 0.0001 . In each run of cross-validation, a multiple linear regression model was fitted in the estimation set with fixed effects of significant markers detected in the association mapping scan. The non-cross-validated accuracy of prediction was determined within the estimation set as square root of the coefficient of determination standardized with the square root of the broad-sense heritability on an entrymean basis (Lande and Thompson, 1990). To determine the cross-validated accuracy of prediction of marker-assisted selection, we estimated the effects for the significant markers using the previous linear regression model fitted with the estimation set and predicted the genotypic value of the lines in the test set. Based on these values, we calculated the cross-validated accuracy of prediction as the Pearson product-moment correlation between predicted and observed genotypic values in the test set standardized with the square root of the broadsense heritability on an entry-mean basis. The average difference between noncross-validated and cross-validated accuracies of prediction was considered as bias. To quantify the proportion of variance explained in the test set because of the exploitation of relatedness, we calculated the prediction accuracy via the same training and test sets as before but using randomly chosen markers. More precisely, in each cross-validation run we randomly chose the same number of markers as the number of QTLs observed in the association mapping scan in the estimation set, estimated the effects for these randomly chosen markers using the estimation set and predicted the genotypic value of the individuals in the test set. The prediction accuracy in the test set was defined as above. The procedure was repeated 1000 times.

\section{Simulation study to examine the impact of relatedness on marker- assisted selection}

We investigated the influence of relatedness on the accuracy of prediction of the phenotypic performance by conducting computer simulations based on the $9 \mathrm{k}$ SNP array data of our study. We selected 30 SNP markers with a linkage disequilibrium measured as $r^{2}$ values of 0.1 or less to all other markers. We then assumed that each of these 30 SNPs is perfectly linked to one QTL explaining $1.7 \%$ of the genotypic variance each, resulting in a total of $50 \%$. We further assumed a heritability of 0.90 . The simulated data set was used to conduct association mapping coupled with fivefold cross-validation with 100 runs, with the accuracy of prediction defined as the Pearson product-moment correlation between predicted and observed genotypic values in the test set standardized with the square root of the heritability. The following four scenarios were contrasted in order to separate the contributions of marker effects and relatedness: (1) we assumed the $30 \mathrm{SNP}$ markers to represent predefined marker-trait associations and estimated their effects based on the estimation set. The accuracy of prediction was validated in the test sets. (2) We assumed that the 30 SNP markers were not predefined and performed an association mapping scan in the estimation set to identify the 30 most significant markertrait associations. Effects of these marker-trait associations were estimated based on the estimation set. The accuracy of prediction was evaluated in the test set. (3) Randomly selected 30 SNP markers excluding those with significant marker-trait associations were sampled and their effects were estimated in the estimation set. The accuracy of prediction was then evaluated in the test set. (4) Randomly selected 30 SNP markers were sampled among all SNPs and their effects were estimated in the estimation set. The accuracy of prediction was then evaluated in the test set.

\section{Genomic selection}

Three genomic selection models were applied in evaluating the prediction accuracy. They are ridge regression best linear unbiased prediction (RR-BLUP; Whittaker et al., 2000; Meuwissen et al., 2001), reproducing kernel Hilbert space regression (RKHSR; Gianola and van Kaam, 2008) and Bayes-C $\pi$ (Habier et al., 2011). In the following, we give some descriptions about the three models. For more details, we refer to the above references.

Let $n$ be the number of genotypes, $m$ be the number of markers and $l$ be the number of environments. The RR-BLUP model has the form $y=1_{n} \mu+X g+e$, where $y$ is the vector of best linear unbiased estimates of FHB scores for all genotypes across environments, $1_{\mathrm{n}}$ denotes the vector of 1 's, $\mu$ is the overall mean, $g$ is the vector of marker effects (for SSR markers, allele effects), $X$ is the corresponding design matrix and $e$ is the residual term.

In the model, we assumed that marker and residual effects are random and follow the multivariate normal distribution $g \sim N\left(0, \sigma_{g}^{2}\right), e \sim N\left(0, \sigma_{e}^{2}\right)$, where $\sigma_{g}^{2}=\sigma_{G}^{2} / m$ for SNP markers and $\sigma_{e}^{2}=\sigma_{R}^{2} / l$. Here $\sigma_{G}^{2}$ and $\sigma_{R}^{2}$ are the genotypic and residual variance components obtained in the mixed model in the phenotypic data analysis. The penalty parameter is $\lambda=\left(\sigma_{R}^{2} / l\right) /\left(\sigma_{G}^{2} / m\right)$. For SSR markers, we treated $\sigma_{G}^{2} / m$ as shared by all alleles in each marker, with each allele effect having the same variance within each marker. Hence, the variances of allele effects are different among markers.

The RKHSR model is of the form $y=1_{n} \mu+K \alpha+e$, where $y, 1_{n}, \mu$ and $e$ are the same as in the RR-BLUP model, $\alpha \sim N\left(0, K^{-1} \sigma_{\alpha}^{2}\right)$ is a vector of random effects and $K$ is the $n \times n$ symmetric positive-definite matrix whose entries are defined by

$$
K_{i j}=\exp \left[\frac{-\left(x_{i}-x_{j}\right)^{\prime}\left(x_{i}-x_{j}\right)}{h}\right] .
$$

In the above formula, $x_{i}$ and $x_{j}$ are $(m \times 1)$ vectors of marker indices for the $i$-th and $j$-th genotype respectively, and $h$ is a smoothing parameter. To determine $h$ and estimate $\sigma_{\alpha}^{2}$, we first chose a grid of values for $h$. For each value of $h$, we estimated $\sigma_{\alpha}^{2}$ using a restricted maximum likelihood approach and then calculated the fitted values of the model. Finally we chose the value $h$ optimizing the generalized cross-validation statistic of the model.

The basic model of Bayes-C $\pi$ is the same as RR-BLUP. However, all parameters are treated as random variables in a Bayesian framework. First, we defined the prior distributions as $g \sim N\left(0, \sigma_{g}^{2}\right), e \sim N\left(0, \sigma_{e}^{2}\right)$. The prior of $\mu$ is a constant. The prior distribution of $\sigma_{g}^{2}$ is assumed to be zero with probability $\pi$ and a scaled inverse chi-squared distribution with probability $(1-\pi)$. The probability $\pi$ is a random variable whose prior distribution is uniform on the interval $[0,1]$. The prior distribution of $\sigma_{e}^{2}$ is also scaled inverse chi-squared. A Gibbs sampler algorithm was then implemented to infer all the parameters in the model. It was run for 10000 cycles and the first 1000 cycles were discarded as burn in. The samples of $g$ from all later cycles were averaged to obtain estimates of the marker effects. 
We evaluated the accuracy of prediction of the three genomic selection models using a fivefold cross-validation across genotype scheme. The individuals were randomly divided into five subsets. Then four of the five subsets were used as the estimation set and the genotypic values of the individuals in the remaining test set were calculated. After the genotypic values of all individuals were calculated, the means and standard errors of the prediction accuracy were calculated using a bootstrap approach (Rutkoski et al., 2012). The sampling of bootstrap was repeated 1000 times. The accuracy of prediction was defined as the correlation between observed and predicted values divided by the square root of the heritability $r_{G S}=\operatorname{cor}\left(y_{\text {pred }}, y_{o b s}\right) / h$.

Moreover, we studied the effect of the number of markers on the prediction accuracy of genomic breeding values through cross-validation. Therefore, we varied in the cross-validation studies the number of markers from 5 to $90 \%$ of markers available for each of the three marker sets (9k and 90k SNP, SSR). All calculations were done using $\mathrm{R}$ and Asreml-R (Gilmour et al., 2006).

\section{RESULTS}

Population structure observed based on SSR markers, 9k SNP and 90 SNP array data

We observed a wide-range of pairwise Rogers' distances for all three marker systems used (Table 1). The coefficient of variation (CV) of pairwise Rogers' distances was similar for the $9 \mathrm{k}(\mathrm{CV}=0.17)$ and $90 \mathrm{k}$ $(\mathrm{CV}=0.15)$ SNP array data, both slightly exceeding that for the SSR marker data $(\mathrm{CV}=0.12)$. The distance matrices estimated with the three different marker data sets were significantly $(P<0.01)$ associated with Pearson product-moment correlation coefficients of above 0.84 .

The analysis of the population structure revealed the absence of genetically distinct sub-populations for all three marker data sets (Figure 1 and data not shown). We applied a bootstrapping procedure and found significant $(P<0.05)$ differences among the SSR and SNP markers in terms of the explained proportion of molecular variation for the first three principal components (Figure 2). This suggested that the population structure estimated based on SSR markers is more complex than that examined with SNP markers. The observed differences among SSR and SNP markers may be attributed to the biallelic (SNPs) and multiallelic (SSRs) nature of the two marker types, respectively.

Artificial inoculation resulted in high-quality phenotypic data Disease pressure was high as reflected by a broad range of FHB scores among the panel of 372 European wheat inbred lines with a mean value of $11 \%$ (Table 2). The distribution of FHB scores of the 358 winter wheat varieties was similar to that of the 14 spring wheat varieties (data not shown), suggesting that combined analyses are not afflicted by population stratification effects. The genetic variance of

Table 1 First- and second-degree statistics for RD among 372 wheat lines estimated by three different sets of markers as well as Pearson product-moment correlation coefficients among distance matrices $\left(r_{R D}\right)$

\begin{tabular}{lccc}
\hline Parameters & $9 k$ SNP & $90 k$ SNP & SSR \\
\hline Mean & 0.332 & 0.326 & 0.502 \\
Range & $0.002-0.504$ & $0.002-0.486$ & $0.052-0.667$ \\
CV (s.e.) & $0.166(0.004)$ & $0.151(0.001)$ & $0.120(0.005)$
\end{tabular}

$r_{R D}$

$\begin{array}{lll}9 k \text { SNP } & 0.90^{a} & 0.84^{a} \\ 90 k \text { SNP } & & 0.85^{a}\end{array}$

Abbreviations: CV, coefficient of variation; RD, Rogers' distances; SNP, single-nucleotide polymorphism; SSR, simple sequence repeat. polymorphism; SSR, simple sequence repeat.
aSignificant at the level of 0.01 in the Mantel test.
FHB scores was significantly $(P<0.01)$ larger than zero. Heritability on the line mean basis was high and amounted to $91 \%$. This clearly underlines the high quality of the phenotyping data.

FHB resistance is often influenced by flowering time and plant height. For our panel of 372 wheat lines, Kollers et al. (2013a) estimated that flowering time explained only $5 \%$ of the variation in FHB resistance. Consequently, we expect only minor relevance of flowering time as masking effect on FHB resistance. Plant height had a slightly higher predictive value explaining $12 \%$ of the variation of FHB resistance. Consequently, it is likely that genes with large impact on plant height have a role for genomics-based prediction of FHB resistance.

\section{Marker-assisted selection for FHB resistance}

The functional markers for $P p d-D 1$ and Rht-D1, two major genes in the regulation of flowering time and plant height in wheat, were significantly $(P<0.005)$ associated with $\mathrm{FHB}$ resistance (Supplementary Table S2). This result could be expected considering the association between FHB resistance and plant height as well as heading time observed at the phenotypic level (Kollers et al., 2013a). The marker-trait association for Rht-B1 was not significant $(P>0.05)$, which could be explained by the low allele frequency of the mutant allele detected in only 26 of the investigated varieties. Another explanation may be given by the known difference in the effects of Rht-B1 and Rht-D1 on FHB (Srinivasachary et al., 2009). The Rht-D1 gene significantly influences both type 1 resistance (resistance to initial infection) and type 2 resistance (resistance to spread of the fungus within the spike), whereas $R h t-B 1$ has significant influence only on type 1 resistance.

We performed more detailed further analyses for the two functional genes $P p d-D 1$ and Rht-D1. Excluding the two markers and closely linked ones from our association study led to a drop of cross-validated prediction accuracy of $14-30 \%$ (depending on the threshold used). In comparison, the cross-validated accuracy of prediction for the corresponding random scenario avoiding sampling the two genes dropped only by $4.2-17 \%$. Thus, $P p d-D 1$ and $R h t-D 1$ are functional in

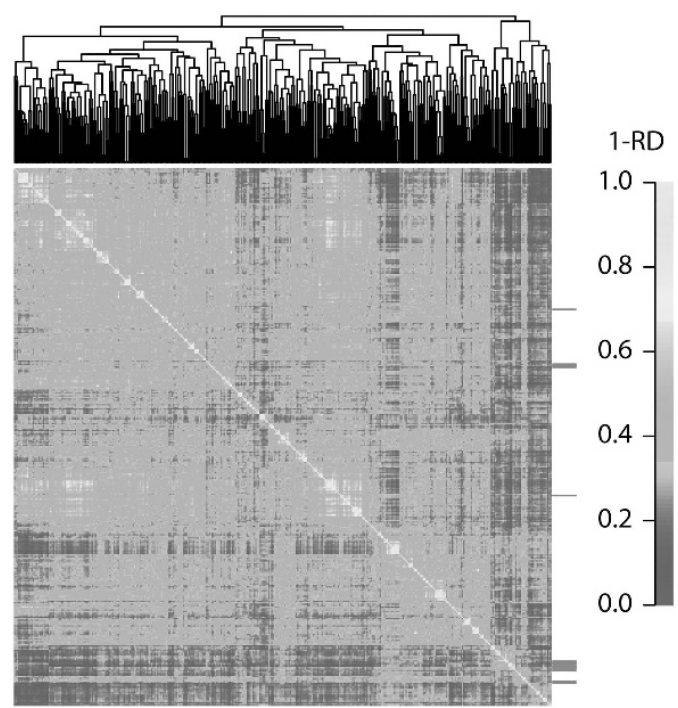

Figure 1 Heat map plot of the relationship measured as one minus Rogers' distance based on the 90k SNP array data of the 372 wheat lines ordered according to a hierarchical cluster analysis. The red segments indicate the positions of the 14 spring varieties in the diversity panel. A full color version of this figure is available at the Heredity journal online. 


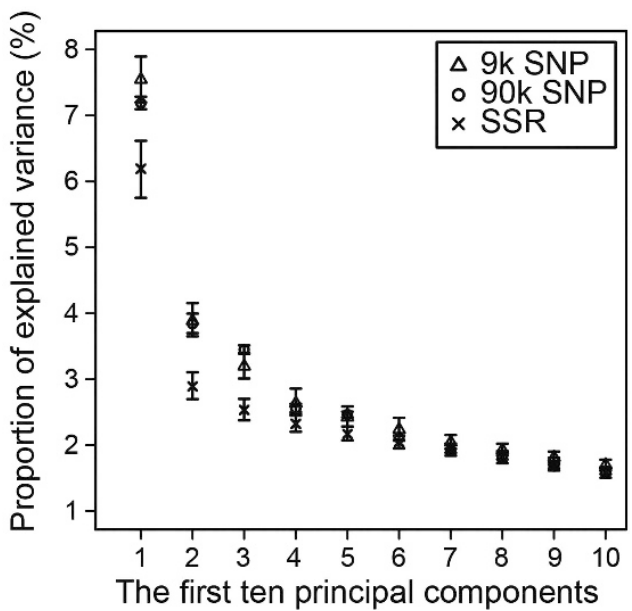

Figure 2 Principal component analyses of the 372 wheat lines based on the three marker sets 9k SNP array, 90k SNP array and SSR markers. Proportions of variance explained by the first 10 principal components are shown with confidence intervals obtained by 1000 bootstrap samples.

Table 2 First- and second-degree statistics for 372 wheat lines evaluated for FHB resistance (\%) in four environments

\begin{tabular}{lc}
\hline Source & Value \\
\hline Mean and range & $11.29(0-59.5)$ \\
Genotypic variance & $42.44^{\mathrm{a}}$ \\
$\mathrm{G} \times \mathrm{E}$ variance & $9.82^{\mathrm{a}}$ \\
Residual variance & $21.73^{\mathrm{a}}$ \\
Heritability $\left(\mathrm{h}^{2}\right)$ & 0.91 \\
\hline
\end{tabular}

Abbreviation: FHB, Fusarium head blight.

The range of the FHB score is from $0 \%$ (the most resistant) to $100 \%$ (the most susceptible). asignificantly different from 0 at the level of 0.01 . determining FHB resistance. When solely $P p d-D 1$ and $R h t-D 1$ markers were used in prediction, the cross-validated accuracy reached 0.50 . However, this result has to be considered with caution, as the correlation between Rogers' distances among the 372 lines estimated based on all markers with the one based on solely Ppd-D1 and Rht-D1 amounted to 0.26 (significant at $P<0.001$ ). Thus, a large part of the accuracy of prediction accuracy was due to relatedness.

In preparation for genome-wide association mapping, qq-plots based on observed $P$-values and expected $P$-values were drawn (Supplementary Figure S2). For all three marker data sets, the plots were close to a diagonal line, confirming that the association mapping model used in this study has adequately controlled false positives because of population structure. A total of 4, 114 and 6 significant $(P<0.005)$ marker-trait associations were detected for the 9k SNP array, 90k SNP array and SSR markers, respectively (Supplementary Table S2). It is worth to note that the effective number of significant marker-trait associations defined as the minimal number of principal components needed to portray $95 \%$ of the variation of the significant markers was 40 for the 90k SNP array, indicating that we indeed detected more marker-trait associations than in the 9k SNP array. We then compared the importance of different parameters for the selection of potential functional markers for use in breeding or map-based cloning activities. When we first tested the deviation of $P$-values of marker-trait associations in fivefold cross-validation runs, we observed substantial variation in $P$-values (Figure 3$). P$-values of markers with minor allele frequencies were on average less robust than those for markers with intermediate frequencies. Nevertheless, also $P$-values for markers with intermediate frequencies were sometimes substantially higher in the full data set in contrast to the estimation sets. Thus, inspecting the distribution of the cross-validated $P$-values in the estimation sets can be used as one criterion in the search of robust candidates such as BS00043676_51 (coded as SNP_2144, see Supplementary Figure S1) for map-based cloning.
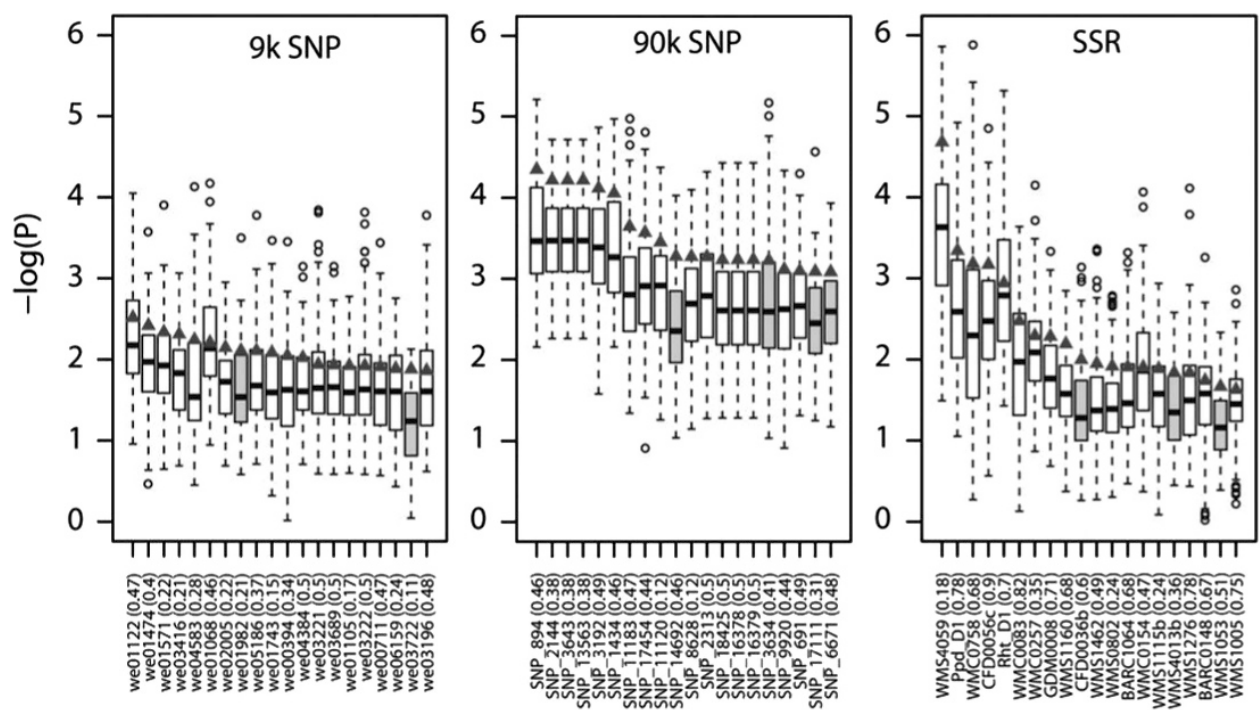

Figure $3 P$-values of 20 most significant markers detected in the genome-wide association mapping scan (red triangles) and the distributions of $P$-values in the 100 cross-validation runs. The gray boxes highlight markers that are not significant according to the median $P$-values of the cross-validation runs. The polymorphic information content (PIC) of each marker is indicated in the bracket after the name of the marker. Linkage disequilibrium among markers is given in Supplementary Figure S1. The names of markers in the 90k SNP chip were coded for better visibility. The original names of those markers are listed in Supplementary Figure S1. A full color version of this figure is available at the Heredity journal online. 
Second, we investigated the correspondence between the observed $P$-values with two approaches to estimate the explained proportion of phenotypic variation. The likelihood-ratio-based parameter $R_{L R}^{2}$ was closely associated with the $P$-values in contrast to the $R^{2}$ estimated with multiple regressions (Figure 4$)$. The strong deviation of $P$-values of the association mapping scan from $R^{2}$ values in a multiple regression is not surprising, because the latter approach ignores information on genetic relatedness. In contrast, the close association between $R_{L R}^{2}$ and $P$-values of the association mapping scan is expected as both are based on similar biometrical models considering genetic relatedness. The different interpretations of $R^{2}$ versus $R_{L R}^{2}$ have to be considered while selecting markers for further use in marker-assisted breeding or map-based cloning. If the distance matrix of functional markers such as Rht-D1 is associated $(r=0.26 ; P<0.001)$ with the overall genetic structure of the mapping population, then $R_{L R}^{2}$ will underestimate the value of true functional markers.

Irrespective of the chosen significance thresholds, the analyses revealed an overestimation of the accuracy of prediction of FHB resistance for all three marker systems as is reflected by higher accuracies observed for non-cross-validated compared with the cross-validated value (Figure 5). This bias was less pronounced for the 9k SNP array compared with the 90k SNP array and SSR marker data analyses. Interestingly, the accuracy of prediction dropped down only slightly when information on marker-trait associations was ignored in cross-validation by using random samples of markers in numbers corresponding to the average number of QTL detected. This suggests that relatedness is the major driving force for the prediction accuracy of marker-assisted selection.

To examine our findings on the role of relatedness in more detail, we performed computer simulations in different scenarios. For all, we postulated an involvement of 30 QTLs, each explaining $\sim 1.7 \%$ of the genotypic variation, summing up to $50 \%$ of the total genotypic variation. In the first simulation scenario, we assumed that all SNPs would have marker-trait associations in order to study how precise QTL effects can be estimated in the underlying mapping population. We observed an accuracy of prediction of 0.64 (Figure 6), which corresponds to $75 \%$ of the genotypic variation explained by the 30 QTLs. In the second scenario, we assumed that marker-trait associations were unknown and had to be detected in a genome-wide association mapping scan. Before calculating the prediction accuracy, we checked the false-positive rate in the scan, which was $0 \%$ under the threshold of $P<0.001$. Thus, population structure was adequately controlled in our association mapping model. The accuracy of

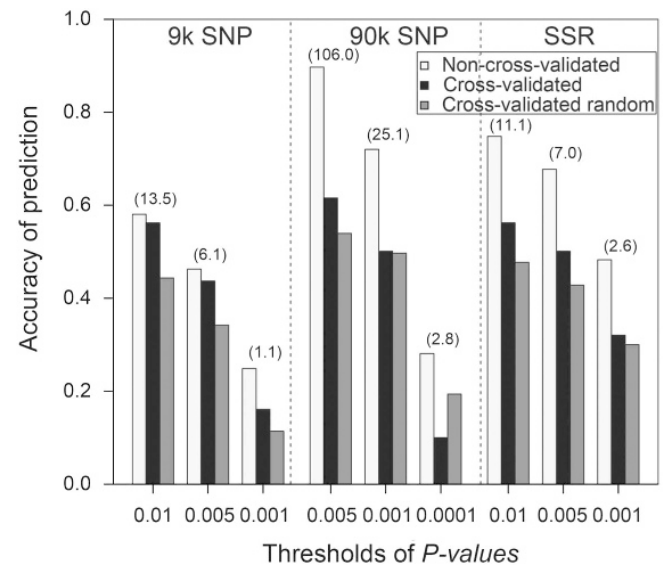

Figure 5 Marker-assisted selection for FHB resistance based on 9k SNP array, 90k SNP array and SSR markers. Accuracy of prediction was determined non-cross-validated (white columns), cross-validated as the Pearson product-moment correlation between predicted and observed genotypic values in the test set (black columns) or cross-validated based on a randomly selected set of markers (gray columns). The average number of significant markers used for prediction is indicated in brackets.
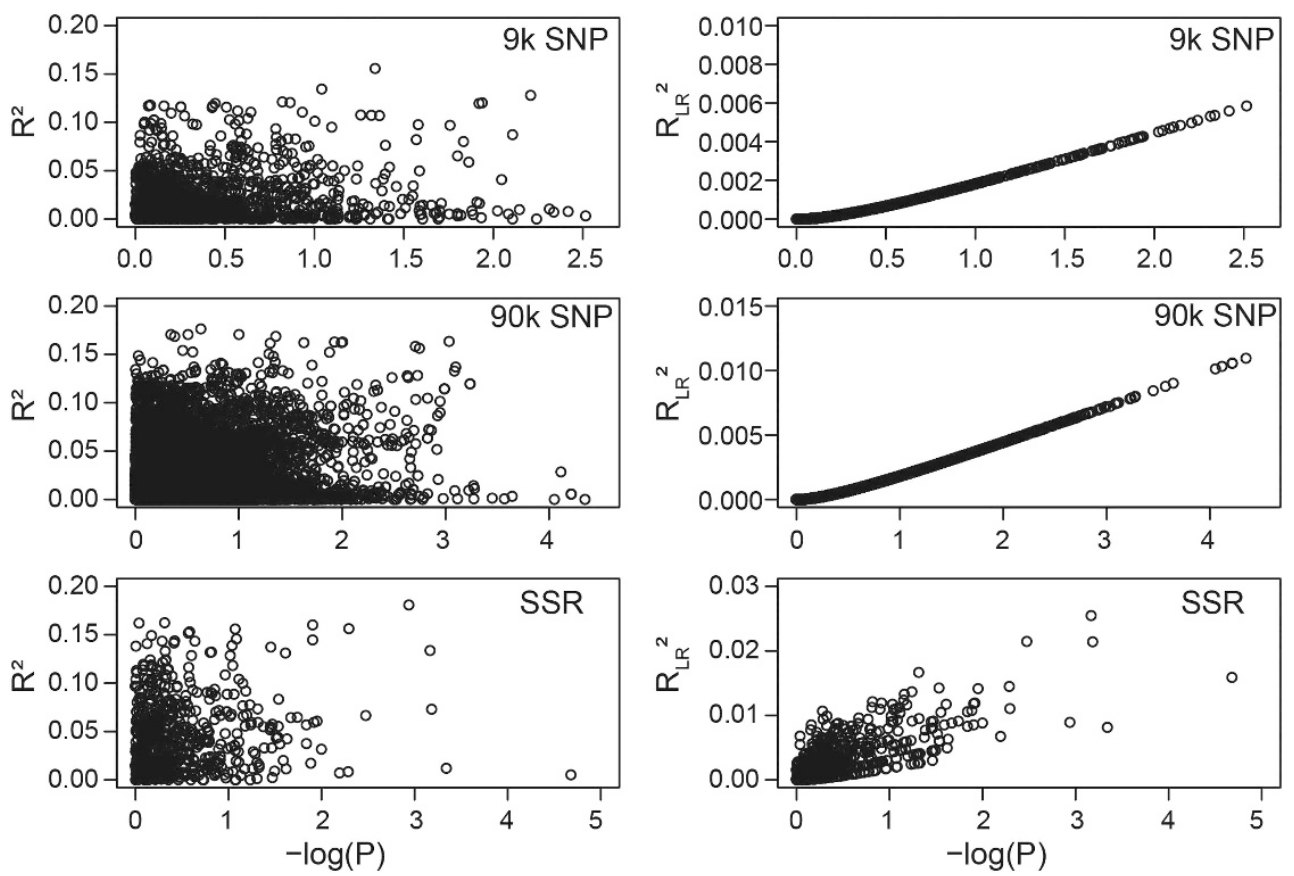

Figure 4 The relationship between $P$-values and the proportion of the phenotypic variance explained by all $Q T L s$ estimated using multiple regressions ( $R^{2}$ ) and a likelihood-ratio-based method $\left(R^{2} L R\right)$. 


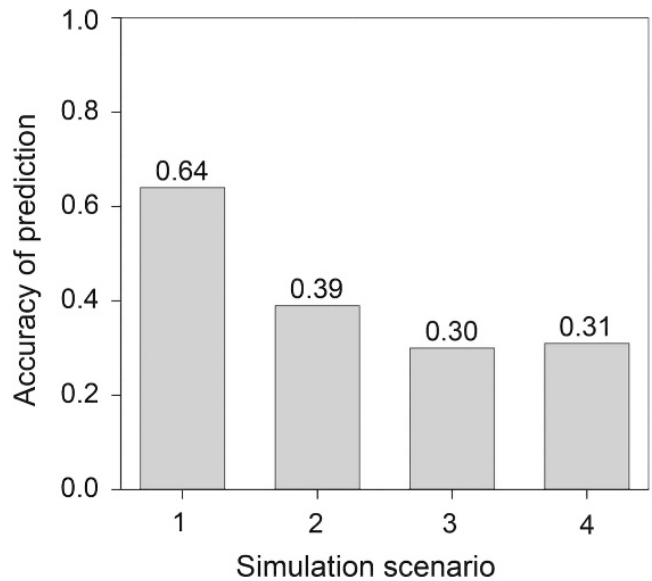

Figure 6 Simulation of marker-assisted selection based on 9k SNP array data to separate contributions of marker effects and relatedness. The crossvalidated accuracy of prediction defined as the Pearson product-moment correlation between predicted and observed genotypic values in the test set. (1) We assumed the 30 SNP markers to represent predefined marker-trait associations and estimated their effects based on the estimation set. (2) We assumed that the 30 SNP markers were not predefined and performed an association mapping scan in the estimation set to identify the 30 most significant marker-trait associations. Effects of these marker-trait associations were estimated based on the estimation set. (3) We randomly selected 30 SNP markers excluding those with significant marker-trait associations, and estimated their effects in the estimation set. (4) We randomly selected 30 SNP markers among all SNPs and estimated their effects in the estimation set.

prediction decreased to 0.39 by approximately $40 \%$ compared with the first scenario, which was nevertheless still surprisingly high taking into consideration the population size and the effect size of each QTL. The QTL detection rate per cross-validation run on average reached only $10 \%$. Contrasting the second scenario with a third scenario using 30 SNP markers unlinked to marker-trait associations led to only approximately $20 \%$ lower accuracy of prediction of 0.30 . This clearly underlines that genetic relatedness is a major driving force for prediction accuracy in marker-assisted selection for complex traits in the absence of QTL with large effect sizes. The fourth scenario was similar to the third one except that we randomly sampled 30 SNPs out of all SNPs, thus potentially selecting also marker-trait associations. This scenario reflected the 'random' marker scenario of the experimental data analyses. We observed only a marginal increase of the accuracy of prediction to 0.31 . Compared with the third scenario, this suggested that the bias in the 'random' scenario of the experimental data analysis by sampling all markers including also putative QTL can be ignored.

\section{Genomic selection of FHB resistance}

The cross-validated accuracy of prediction of phenotypic performance was at a significance threshold of $P<0.005$ on average $47 \%$ higher for genomic than marker-assisted selection across 9k SNP array, 90k SNP array and SSR marker-based data (Table 3 and Figure 5). Interestingly, we observed no significant differences in the accuracies of prediction between marker platforms. The mean accuracies of prediction also did not differ significantly across the three genomic selection models RRBLUP, RKHSR and Bayes-C $\pi$. Re-sampling of reduced sets of markers revealed that the accuracy of prediction was already reaching a plateau at rather low marker densities (Figure 7).
Table 3 Means and s.e. of cross-validated prediction accuracies of genomic selection for Fusarium head blight resistance of 372 wheat lines based on the three different marker sets 9k SNP array, 90k SNP array and SSR markers and the three different biometrical models RR-BLUP, RKHSR and Bayes-C $\pi$

\begin{tabular}{lccc}
\hline Marker set & RR-BLUP & RKHSR & Bayes-C $\pi$ \\
\hline 9K SNP & $0.706 \pm 0.032$ & $0.724 \pm 0.033$ & $0.716 \pm 0.031$ \\
90K SNP & $0.740 \pm 0.031$ & $0.742 \pm 0.036$ & $0.721 \pm 0.032$ \\
SSR & $0.710 \pm 0.034$ & $0.713 \pm 0.035$ & $0.684 \pm 0.037$ \\
\hline
\end{tabular}

Abbreviations: RKHSR, reproducing Kernel Hilbert space regression; RR-BLUP, ridge regression best linear unbiased prediction; SNP, single-nucleotide polymorphism; SSR, simple sequence repeat.

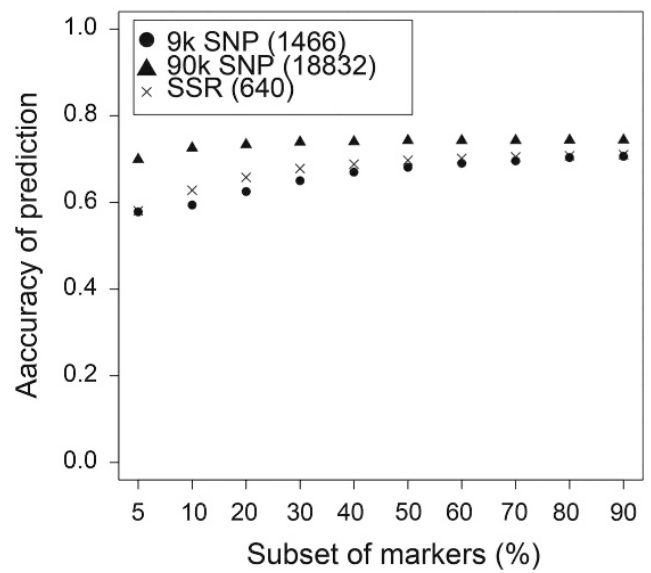

Figure 7 Effect of the number of markers on the prediction accuracy of genomic selection. The total number of markers used is indicated in brackets.

\section{DISCUSSION}

Relatedness strongly impacts the cross-validated prediction accuracy in association mapping studies

It has been suggested that high-throughput genome-wide association mapping studies have the potential to simultaneously answer two central questions in quantitative genetics, regarding (I) how many genes influence a particular trait and (II) what the allele distributions at these gene loci are (Wallace et al., 2013). For complex traits such as FHB resistance that are determined by a rather large number of genes, association mapping studies in wheat were often found to explain a surprisingly high amount of the genetic variation (Miedaner et al., 2011; Rutkoski et al., 2012; Kollers et al., 2013a). Our results are in accordance with these previous findings, as the marker-assisted selection approaches based on the three marker systems explained up to $78 \%$ of the phenotypic variation for the total population when a significance threshold of $P<0.005$ was applied. This number dropped in fivefold cross-validation (Figure 5) as was expected from previous results (Guo et al., 2012; Miedaner et al., 2013; Zhao et al., 2013; Gowda et al., 2014; Würschum and Kraft, 2014). Nevertheless, the cross-validated accuracy of prediction of FHB resistance is still high and in most cases higher than using randomly sampled markers (Figure 5), suggesting that genome-wide association mapping studies indeed have the potential to at least partially answer the two central questions on the genetic architecture of FHB resistance outlined above.

In order to maximize the information gained from a genome-wide mapping study, an optimum significance threshold reflecting a balance 
between false-discovery rate and power to detect QTL of interest needs to be chosen (Moreau et al., 1998). Applying increasingly conservative thresholds, we observed a monotonic decreasing trend in crossvalidated values of prediction accuracies, which suggests that within the investigated range of $P$-values, increasing the power of QTL detection with a more relaxed significance threshold was more relevant than increasing the risk to detect false-positive QTL. However, a further explanation for this is that relatedness also contributes to the accuracy of prediction of marker-assisted selection. Every sequence polymorphism, also the ones defining QTL, will contribute to relatedness. This relatedness is then exploited in marker-assisted selection. This has been observed earlier in a genome-wide mapping study in a vast hybrid wheat population (Gowda et al., 2014) and in two bi-parental rye populations (Wang et al., 2014b). Relatedness can be portrayed more precisely with relaxed significance thresholds that allow a high number of SNPs to be used in the prediction models.

We also sampled a random set of markers corresponding to the number of QTL detected in the QTL mapping scans, and used this random set of markers to predict the phenotypic performance by applying fivefold cross-validation. Our finding that using significant marker-trait associations did lead to only marginal higher accuracy of prediction of FHB resistance in comparison with the random set of markers was surprising (Figure 5). It clearly indicated that the contribution of genetic relatedness to the prediction ability of significant markers is far from negligible. To confirm that, we compared the correlations between Rogers' distances among the 372 lines estimated using full marker data set and that based on significant markers detected in the association mapping scan. We observed significant correlations that amounted to $0.28(P<0.001)$ with only 4 significant SSR markers and $0.24(P<0.001)$ with 21 significant SNP markers in the $9 \mathrm{k}$ array. Hence, our analysis underlined that genetic relatedness has a key role in the prediction of FHB resistance in marker-assisted selection. We further substantiated our results applying simulations (Figure 6). As a consequence, relatedness must be taken into account when interpreting the results on the genetic architecture of complex traits in genome-wide association mapping studies.

Influence of marker system and biometrical model on the accuracy of prediction in genomic selection

The non-linear, semi-parametric RKHSR has the potential to capture complex interactions better than RR-BLUP and Bayes-C $\pi$ assuming linear relationships between phenotype and genotype (de los Campos et al., 2010). Previous genome-wide association mapping studies suggested presence of epistatic effects for FHB resistance (Miedaner et al., 2012). Thus, it is tempting to speculate that RKHSR outperforms RR-BLUP and Bayes-C $\pi$ as observed in several previous genomic selection studies (Crossa et al., 2010, 2011; de los Campos et al., 2010; Heslot et al., 2012). In contrast to this expectation, however, no significant differences among the accuracies of the three applied genomic selection models were observed (Table 3). The variation of the accuracy of prediction of the applied genomic selection models across the different cross-validation scenarios also did not differ. Consequently, RR-BLUP, the model with the lowest requirement on computational time, seems to be the most promising model for implementation of genomic selection for FHB resistance in wheat breeding programs.

Genomic selection exploits to a large extent the relatedness among the plant material in estimation and test sets to predict the phenotypic performance. In total, $29 \%$ of the variation in Rogers' distances observed with the 90k SNP array cannot be explained with the SSR marker data (Table 1). Thus, the choice of the marker set is expected to impact the accuracy of genomic selection. In addition, the bootstrap analyses of the principal component analyses revealed a more complex population structure for the SSR as compared with the SNP arrays (Figure 2). This slightly different picture is expected because of the different nature of SNP versus SSR markers, with SSR markers possessing a higher resolution to unravel the more recent population history (Würschum et al., 2013). Consequently, it is surprising that we did not observe differences in accuracies of prediction of the different marker systems. One possible explanation for the similar accuracies of prediction of the different marker systems is that the true relationship matrix for the 372 wheat lines lies in between the two matrices estimated with the SSR markers or the two SNP arrays. This would lead to similar accuracies of prediction of SSR and SNP marker data.

\section{CONCLUSIONS}

Our cross-validation study clearly underlined that the accuracy of prediction of marker-assisted selection is strongly influenced by relatedness. This was confirmed applying computer simulations. Our findings, however, also showed that relatedness can be better exploited by applying genomic selection instead of marker-assisted selection. Marker density had only a marginal impact on the prediction accuracy of genomic selection. Consequently, genomic selection as an attractive complementation to phenotypic selection of FHB resistance can be most efficiently implemented based on low- to medium-density marker panels.

\section{DATA ARCHIVING}

Relevant data sets have been added as Supplementary Tables S1 and S2.

\section{CONFLICT OF INTEREST}

SK, VK and EE are employed by the company KWS LOCHOW GMBH. OA and MH are employed by Syngenta Seeds and MWG and JP are employed by the company TraitGenetics GmbH. The companies have commercial interest in the results for application in variety development and for the provision of genotyping services. This does not alter the authors' adherence to all HEREDITY policies on sharing data and materials.

\section{ACKNOWLEDGEMENTS}

The phenotypic and genotyping data were generated within the frames of the projects GABI-Wheat and VALID (project numbers 0315067 and 0315947) funded by the Plant Biotechnology program of the German Federal Ministry of Education and Research (BMBF).

Akbari M, Wenzl P, Caig V, Carling J, Xia L, Yang S et al. (2006). Diversity arrays technology (DArT) for high-throughput profiling of the hexaploid wheat genome. Theor Appl Genet 113: 1409-1420.

Barrett BA, Kidwell KK (1998). AFLP-based genetic diversity assessment among wheat cultivars from the Pacific Northwest. Crop Sci 38: 1261-1271.

Beales J, Turner A, Griffiths S, Snape J, Laurie DA (2007). A pseudo-response regulator is misexpressed in the photoperiod insensitive Ppd-Dla mutant of wheat (Triticum aestivum L.). Theor App/ Genet 115: 721-733.

Becher R, Miedaner T, Wirsel SG (2013). 8 Biology, diversity, and management of FHBcausing fusarium species in small-grain cereals. In: Frank $\mathrm{K}$ (ed.) Agricultural Applications. Springer: Berlin, Heidelberg. pp 199-241.

Bernardo AN, Ma H, Zhang D, Bai G (2012). Single nucleotide polymorphism in wheat chromosome region harboring Fhb1 for Fusarium head blight resistance. Mol Breed 29: 477-488.

Buerstmayr H, Lemmens M, Schmolke M, Zimmermann G, Hartl L, Mascher F et al. (2008). Multi-environment evaluation of level and stability of FHB resistance among 
parental lines and selected offspring derived from several European winter wheat mapping populations. Plant Breed 127: 325-332.

Buerstmayr H, Ban T, Anderson JA (2009). QTL mapping and marker-assisted selection for Fusarium head blight resistance in wheat: a review. Plant Breed 128: 1-26.

Cavanagh CR, Chao S, Wang S, Huang BE, Stephen S, Kiani S et al. (2013). Genome-wide comparative diversity uncovers multiple targets of selection for improvement in hexaploid wheat landraces and cultivars. Proc Nat Acad Sci USA 110: 8057-8062.

Chao S, Sharp PJ, Worland AJ, Warham EJ, Koebner RMD, Gale M D (1989). RFLP-based genetic maps of wheat homoeologous group 7 chromosomes. Theor Appl Genet 78: 495-504.

Crossa J, de Los Campos G, Pérez P, Gianola D, Burgueño J, Araus JL et al. (2010) Prediction of genetic values of quantitative traits in plant breeding using pedigree and molecular markers. Genetics 186: 713-724.

Crossa J, Pérez P, de los Campos G, Mahuku G, Dreisigacker S, Magorokosho C (2011). Genomic selection and prediction in plant breeding. J Crop Improv 25: 239-261.

de los Campos G, Gianola D, Rosa GJ, Weigel KA, Crossa J (2010). Semi-parametric genomic-enabled prediction of genetic values using reproducing kernel Hilbert spaces methods. Genet Res 92: 295-308.

Ellis MH, Spielmeyer W, Gale KR, Rebetzke GJ, Richards RA (2002). "Perfect" markers for the Rht-B1b and Rht-D1b dwarfing genes in wheat. Theor Appl Genet 105: 1038-1042.

Gianola D, van Kaam JB (2008). Reproducing kernel Hilbert spaces regression methods for genomic assisted prediction of quantitative traits. Genetics 178: 2289-2303.

Gilmour AR, Gogel BJ, Cullis BR, Thompson R (2006). ASReml user guide release 2.0 VSN International Ltd.

Gowda M, Zhao Y, Würschum T, Longin CF, Miedaner T, Ebmeyer E et al. (2014). Relatedness severely impacts accuracy of marker-assisted selection for disease resistance in hybrid wheat. Heredity 112: 552-561.

Guo Z, Tucker DM, Lu J, Kishore V, Gay G (2012). Evaluation of genome-wide selection efficiency in maize nested association mapping populations. Theor App/ Genet 124 261-275.

Habier D, Fernando RL, Kizilkaya K, Garrick DJ (2011). Extension of the Bayesian alphabet for genomic selection. BMC Bioinformatics 12: 186

Heslot N, Yang HP, Sorrells ME, Jannink JL (2012). Genomic selection in plant breeding: a comparison of models. Crop Sci 52: 146-160.

Heslot N, Rutkoski J, Poland J, Jannink JL, Sorrells ME (2013). Impact of marker ascertainment bias on genomic selection accuracy and estimates of genetic diversity. PloS One 8: e74612.

Kollers S, Rodemann B, Ling J, Korzun V, Ebmeyer E, Argillier O et al. (2013a). Whole genome association mapping of Fusarium head blight resistance in European winte wheat (Triticum aestivum L.). PLOS ONE 8: e57500.

Kollers S, Rodemann B, Ling J, Korzun V, Ebmeyer E, Argillier O et al. (2013b). Genetic architecture of resistance to Septoria tritici blotch (Mycosphaerella graminicola) in European winter wheat. Mol Breed 32: 411-423.

Kollers S, Rodemann B, Ling J, Korzun V, Ebmeyer E, Argillier 0 et al. (2014). Genomewide association mapping of tan spot reistance (Pyrenophora tritici-repentis) in European winter wheat. Mol Breed 34: 363-371.

Lande R, Thompson R (1990). Efficiency of marker-assisted selection in the improvement of quantitative traits. Genetics 124: 743-756.

Löffler M, Schön CC, Miedaner T (2009). Revealing the genetic architecture of FHB resistance in hexaploid wheat (Triticum aestivum L.) by QTL meta-analysis. Mol Breed 23: 473-488.

Mantel N (1967). The detection of disease clustering and a generalized regression approach. Cancer Res 27: 209-220.

Meuwissen THE, Hayes BJ, Goddard ME (2001). Prediction of total genetic value using genome-wide dense marker maps. Genetics 157: 1819-1829.

Miedaner T, Schneider B, Heinrich N (2002). Reducing deoxynivalenol (DON) accumulation in rye, wheat, and triticale by selection for Fusarium head blight resistance. J App / Genet A 43: 303-310.

Miedaner T, Wilde F, Korzun V, Ebmeyer E, Schmolke M, Hartl L et al. (2009). Marker selection for Fusarium head blight resistance based on quantitative trait loci (QTL) from two European sources compared to phenotypic selection in winter wheat. Euphytica 166: 219-227.
Miedaner T, Würschum T, Maurer HP, Korzun V, Ebmeyer E, Reif JC (2011). Association mapping for Fusarium head blight resistance in European soft winter wheat. Mol Breed 28: 647-655.

Miedaner T, Korzun V (2012). Marker-assisted selection for disease resistance in wheat and barley breeding. Phytopathology 102: 560-566.

Miedaner T, Risser P, Paillard S, Schnurbusch T, Keller B, Hartl L et al. (2012). Broadspectrum resistance loci for three quantitatively inherited diseases in two winter wheat populations. Mol Breed 29: 731-742.

Miedaner T, Zhao Y, Gowda M, Longin CF, Korzun V, Ebmeyer E et al. (2013). Genetic architecture of resistance to Septoria tritici blotch in European wheat. BMC Genomics 14: 858.

Moreau L, Charcosset A, Hospital F, Gallais A (1998). Marker-assisted selection efficiency in populations of finite size. Genetics 148: 1353-1365.

Pestka JJ, Smolinski AT (2005). Deoxynivalenol: toxicology and potential effects on humans. J Toxicol Environ Health B 8: 39-69.

Poland JA, Brown PJ, Sorrells ME, Jannink JL (2012). Development of high-density genetic maps for barley and wheat using a novel two-enzyme genotyping-by-sequencing approach. PLoS ONE 7: e32253.

Röder MS, Korzun V, Wendehake K, Plaschke J, Tixier MH, Leroy P et al. (1998) A microsatellite map of wheat. Genetics 149: 2007-2023.

Rutkoski J, Benson J, Jia Y, Brown-Guedira G, Jannink JL, Sorrells M (2012). Evaluation of genomic prediction methods for Fusarium head blight resistance in wheat. Plant Gen 5: 51-61.

Sneller CH, Paul P, Guttieri M (2010). Characterization of resistance to Fusarium head blight in an eastern US soft red winter wheat population. Crop Sci 50: 123-133.

Srinivasachary S, Gosman N, Steed A, Hollins TW, Bayles R, Jennings P et al. (2009). Semi-dwarfing Rht-B1 and Rht-D1 loci of wheat differ significantly in their influence on resistance to Fusarium head blight. Theor Appl Genet 118: 695-702.

Sun G, Zhu C, Kramer MH, Yang SS, Song W, Piepho HP et al. (2010). Variation explained in mixed-model association mapping. Heredity 105: 333-340.

Utz HF, Melchinger AE, Schön CC (2000). Bias and sampling error of the estimated proportion of genotypic variance explained by quantitative trait loci determined from experimental data in maize using cross validation and validation with independent samples. Genetics 154: 1839-1849.

Verstraete $F$ (2008). European Union legislation on mycotoxins in food and feed. Overview of the decision-making process and recent and future developments. In: Leslie JF, Bandyopadhyay D, Visconti A (eds) Mycotoxins: Detection Methods, Management Public Health and Agricultural Trade. CAB International: Wallingford, UK pp 77-99.

Wallace JG, Larsson SJ, Buckler ES (2013). Entering the second century of maize quantitative genetics. Heredity 112: 30-38.

Wang S, Wong D, Forrest K, Allen A, Chao S, Huang B et al. (2014a). Characterization of polyploid wheat genomic diversity using a high-density 90000 SNP array. Plant Biotechnol J 12: 787-796.

Wang Y, Mette MF, Miedaner T, Gottwald M, Wilde P, Reif JC et al. (2014b). The accuracy of prediction of genomic selection in elite hybrid rye populations surpasses the accuracy of marker-assisted selection and is equally augmented by multiple field evaluation locations and test years. BMC Genomics 15: 556.

Whittaker JC, Thompson R, Denham MC (2000). Marker-assisted selection using ridge regression. Genet Res 75: 249-252.

Würschum T, Langer SM, Longin CFH, Korzun V, Akhunov E, Ebmeyer E et al. (2013). Population structure, genetic diversity and linkage disequilibrium in elite winter wheat assessed with SNP and SSR markers. Theor Appl Genet 126: 1477-1486.

Würschum T, Kraft T (2014). Cross-validation in association mapping and its relevance for the estimation of QTL parameters of complex traits. Heredity 112: 463-468.

Yu J, Pressoir G, Briggs WH, Bi IV, Yamasaki M, Doebley JF et al. (2006). A unified mixedmodel method for association mapping that accounts for multiple levels of relatedness. Nat Genet 38: 203-208

Zhao Y, Gowda M, Würschum T, Longin CFH, Korzun V, Kollers S et al. (2013). Dissecting the genetic architecture of frost tolerance in Central European winter wheat. J Exp Bot 64: 4453-4460.

Zhao Y, Mette MF, Gowda M, Longin CFH, Reif JC (2014). Bridging the gap between marker-assisted and genomic selection of heading time and plant height in hybrid wheat. Heredity 112: 638-645.

Supplementary Information accompanies this paper on Heredity website (http://www.nature.com/hdy) 